\title{
Th1 Pathway: The Missing Link Between Inflammatory Bowel Disease and Microscopic Colitis?
}

\author{
Anna Carrasco ${ }^{1,2} \cdot$ Fernando Fernández-Bañares ${ }^{1,2}$
}

Published online: 3 August 2017

(C) Springer Science+Business Media, LLC 2017

\section{Introduction}

Microscopic colitis (MC) is associated with chronic or intermittent non-bloody watery diarrhea. Its two principal manifestations are differentiated by their histopathological features: collagenous colitis (CC) and lymphocytic colitis (LC). Advancing age, female sex, smoking, and the use of drugs such as proton pump inhibitors and nonsteroidal antiinflammatory drugs are recognized risk factors for the development of MC [1]. Although studies of its natural history and long-term outcome are limited and scarce, it is generally considered a benign condition, and for all practical purposes, MC mostly differs from conventional inflammatory bowel disease (IBD) (i.e., Crohn's disease and ulcerative colitis).

\section{Linkage Between IBD and MC}

Scattered reports, mainly in the form of case reports or small cohort studies, have suggested the possibility that IBD may either "revert" into MC or vice versa, MC could "progress" to IBD, even considering MC as a milder or gentler form of IBD. The largest retrospective cohort

Anna Carrasco

anna.carrasco.garcia@gmail.com

Fernando Fernández-Bañares

ffbanares@mutuaterrassa.es

1 Department of Gastroenterology, Hospital Universitari Mútua Terrassa, Fundació Recerca Mútua Terrassa,

Terrassa, Barcelona, Spain

2 Centro de Investigación Biomédica en Red de Enfermedades Hepáticas y Digestivas (CIBERehd), Madrid, Spain population study conducted to date did not report any association between MC and IBD [2]. What is more, one prospective population-based cohort study, conducted by Thörn et al. [3], specifically aimed to address the potential bidirectional relationship between IBD and MC, including all new diagnoses of both IBD and MC during 2005-2009, also failed to document any significant correlation between the two conditions.

In this issue of Digestive Diseases and Sciences, Li et al. [4] address this controversial topic. In an extensive retrospective study of all diagnosed cases of MC over a 14-year period in a Canadian city, they found 2324 patients diagnosed with $\mathrm{MC}$ and, from those, $\sim 15 \%$ with proven histological outcome were classified into four groups: "good responders" for those with proven histological remission after treatment, "poor responders," with persistent histological lesions after medical treatment, "IBD-regressors," patients with a previous history of IBD and a subsequent diagnosis of $\mathrm{MC}$, and "IBD-progressors" for those who were diagnosed with IBD after the first MC diagnosis. As regards the "good" and "poor" responder groups, only histological criteria were taken into account, despite current guidelines that state that the primary goal in active MC is to achieve clinical remission, as the usefulness of monitoring histological mucosal remission is currently unknown [5].

After sorting patients in the aforementioned groups, 24 out of $2324(1 \%)$ patients were classified as "IBD-regressors" and 20 out of $2324(0.8 \%)$ were classified as "IBDprogressors" [4]. These figures are in agreement with two Swedish retrospective reports by Mellander et al. [6] or Bohr et al. [7] who reported that 2.1 and $1.8 \%$ of MC patients had concomitant or delayed IBD and MC diagnoses, respectively. Along the same line, five out of 790 IBD patients $(0.6 \%)$ developed MC after the IBD diagnosis 
in the IBD Cohort of Uppsala Health Region (ICURE) study [8]. Interestingly, two of the five patients with MC diagnosed after the IBD diagnosis in the ICURE cohort were also affected by celiac disease [8]. Therefore, it remains to be established whether there is a demonstrable pathophysiological mechanism that links IBD and MC or if it is mere association. Perhaps, a weakened mucosal immune system already insulted by one intestinal disease is more prone to react to another stimuli and develop a second intestinal disease. In support of this latter assumption, the authors of a retrospective cohort study including nearly 200 LC patients reported that $12 \%$ of patients had first- or second-degree relatives affected with a digestive disease different than LC (either IBD, CC, or celiac disease) [9], suggesting that an underlying common mucosal abnormality predisposing to digestive inflammatory disorders (MC, IBD or celiac disease) might exist.

The possibility of an initial misdiagnosis of IBD in the "IBD-progressor" group or a later misdiagnosis of $\mathrm{MC}$ in the "IBD-regressor" group in the study by $\mathrm{Li}$ et al. [4] cannot be completely excluded, as the authors acknowledge. It has to be taken into account that up to $35 \%$ of patients with confirmed MC can manifest subtle endoscopic features, such as erythema, edema, or abnormal vessel pattern, mimicking mild IBD [6], and that the presence of histological features characteristic of IBD, such as Paneth cell metaplasia or crypt architectural distortion, is not uncommon in MC patients [10]. Colonic biopsies from inactive Crohn's disease patients are often characterized as focal, patchy, non-specific inflammatory lesions that could be incorrectly interpreted as MC, especially if the number of biopsies and bowel segments analyzed is small [11]. Many IBD-related and non-IBD-related colitides can also manifest with colonic epithelial lymphocytosis without fulfilling LC criteria [12]. Therefore, the differential diagnosis at the clinical, endoscopic, and histopathological levels can sometimes be complicated and misleading.

\section{Increased Th1 Markers in "MC to IBD- Transformers"}

The authors then evaluated several $\mathrm{T}$ cell markers by means of immunohistochemistry in a selected group of patients from the two extreme groups: "good responders" and "IBD-transformers." "IBD-transformers" showed remarkably higher expression of tumor necrosis factor (TNF)- $\alpha$, interferon (IFN)- $\gamma$, and the Th1-specific transcription factor T-bet as compared with MC-resolved cases [4], all such markers suggestive of a Th1 signature profile. Indeed, other studies demonstrated that IFN- $\gamma$ gene expression correlated with the clinical severity (measured as number of stools per day) of MC patients [13, 14]. Those results highlight an interesting issue: $\mathrm{MC}$ patients with increased Th1 expression might have a more severe clinical course associated with severe inflammation. The majority of the studies evaluating the Th1 profile in the MC mucosa are limited to single-staining immunohistochemistry or real-time polymerase chain reaction (PCR) analysis, bulk analyses that do not extend to the single-cell level. In this sense, our reports that measured Th1 and Th17 cells by flow cytometry found that MC patients (both CC and LC subtypes with a mild or benign outcome) had substantially reduced amounts of Th1 and Th17 cells as compared with controls [14] or IBD patients [15], despite increased gene expression of IFN- $\gamma$ and interleukin (IL)-17. Our data suggested that MC is an attenuated form of inflammation, with pro-inflammatory Th1 and Th17 activated at the gene expression levels but blockaded at the protein or cellular level, in a mechanism perhaps mediated by the anti-inflammatory cytokine IL-10 [14]. Considering the results by Li et al. [4], anti-inflammatory mechanisms might fail in some MC patients, enabling Th1 cells to spread, promoting the development of "classical" IBD. Henceforth, the measurement of Th1/IFN- $\gamma$ producer cells might be an ideal biomarker candidate for the assessment of disease severity and long-term outcome for MC patients. Further prospective studies with long-term follow-up should be conducted to support this hypothesis.

Interestingly, this study also demonstrates that "Crohn's disease-progressors" have a significantly higher expression of T-bet compared to "Ulcerative colitis-progressors." In this sense, evaluation of Th1 and Th17 cells with flow cytometry has demonstrated a Th1/Th17 pattern in Crohn's mucosa and a solely Th17 (with total absence of Th1 cells) in ulcerative colitis [15]. Meticulous evaluation of mucosal biomarkers at an early stage might be useful, to predict not only which MC patients are prone to develop IBD, but also in which phenotype.

Overall, results published by Li et al. [4] point toward a dysregulation of the IFN/T-bet axis as trigger or aggravator of mucosal inflammation in MC that could eventually promote the conversion from MC to "classical" IBD.

\section{Future Directions}

Currently, evidence supporting that $\mathrm{MC}$ is indeed a minor or attenuated form of IBD is scarce. Well-designed, population-based prospective studies with clear clinical, endoscopic, and histological diagnostic criteria are therefore essential to corroborate the hypothetical bidirectional link between IBD and MC. This study from Li et al. [4] somewhat clarifies this controversial topic, proposing that an increase in IFN/T-bet-positive cells could be part of the 
immune mucosal pathological pathway, leading to an increased susceptibility to develop IBD among $\mathrm{MC}$ patients.

\section{Compliance of ethical standards}

Conflict of interest The authors declare no conflict of interest.

\section{References}

1. Fernandez-Banares F, Casanova MJ, Arguedas Y, et al. Current concepts on microscopic colitis: evidence-based statements and recommendations of the Spanish Microscopic Colitis Group. Aliment Pharmacol Ther. 2016;43:400-426.

2. Kao KT, Pedraza BA, McClune AC, et al. Microscopic colitis: a large retrospective analysis from a health maintenance organization experience. World J Gastroenterol. 2009;15:3122-3127.

3. Thorn M, Sjoberg D, Ekbom A, et al. Microscopic colitis in Uppsala health region, a population-based prospective study 2005-2009. Scand J Gastroenterol. 2013;48:825-830.

4. Li J, Yan Y, Meng Z, et al. Microscopic colitis evolved into inflammatory bowel diseases is characterized by increased Th1/ Tc1 cells in colonic mucosal lamina propria. Dig Dis Sci. (Epub ahead of print). doi:10.1007/s10620-017-4636-5.

5. Munch A, Aust D, Bohr J, et al. Microscopic colitis: Current status, present and future challenges: statements of the European Microscopic Colitis Group. J Crohns Colitis. 2012;6:932-945.

6. Mellander MR, Ekbom A, Hultcrantz R, Lofberg R, Ost A, Bjork J. Microscopic colitis: a descriptive clinical cohort study of 795 patients with collagenous and lymphocytic colitis. Scand J Gastroenterol. 2016;51:556-562.
7. Bohr J, Tysk C, Eriksson S, Abrahamsson H, Jarnerot G. Collagenous colitis: a retrospective study of clinical presentation and treatment in 163 patients. Gut. 1996;39:846-851.

8. Ronnblom A, Holmstrom T, Tanghoj H, Wanders A, Sjoberg D. Celiac disease, collagenous sprue and microscopic colitis in IBD. Observations from a population-based cohort of IBD (ICURE). Scand J Gastroenterol. 2015;50:1234-1240.

9. Olesen M, Eriksson S, Bohr J, Jarnerot G, Tysk C. Lymphocytic colitis: a retrospective clinical study of 199 Swedish patients. Gut. 2004;53:536-541.

10. Ayata G, Ithamukkala S, Sapp H, et al. Prevalence and significance of inflammatory bowel disease-like morphologic features in collagenous and lymphocytic colitis. Am J Surg Pathol. 2002;26:1414-1423.

11. Goldstein NS, Gyorfi T. Focal lymphocytic colitis and collagenous colitis: patterns of Crohn's colitis? Am J Surg Pathol. 1999;23:1075-1081.

12. Wang N, Dumot JA, Achkar E, Easley KA, Petras RE, Goldblum JR. Colonic epithelial lymphocytosis without a thickened subepithelial collagen table: a clinicopathologic study of 40 cases supporting a heterogeneous entity. Am J Surg Pathol. 1999;23:1068-1074.

13. Kumawat AK, Strid H, Tysk C, Bohr J, Hornquist EH. Microscopic colitis patients demonstrate a mixed Th17/Tc17 and Th1/ Tc1 mucosal cytokine profile. Mol Immunol. 2013;55:355-364.

14. Carrasco A, Esteve M, Salas A, et al. Immunological differences between lymphocytic and collagenous colitis. J Crohns Colitis. 2016;10:1055-1066.

15. Carrasco A, Fernandez-Banares F, Pedrosa E, et al. Regional specialisation of $\mathrm{T}$ cell subsets and apoptosis in the human gut mucosa: differences between ileum and colon in healthy intestine and inflammatory bowel diseases. $J$ Crohns Colitis. 2016;10:1042-1054. 